\title{
AVES ACUÁTICAS EN EL HUMEDAL URBANO DE TRES PUENTES, PUNTA ARENAS (53 S), CHILE AUSTRAL.
}

\author{
WATERBIRDS IN THE URBAN WETLAND TRES PUENTES, \\ PUNTA ARENAS CITY (53 S), AUSTRAL CHILE.
}

Alejandro Kusch¹, Jaime Cárcamo ${ }^{2,3} \&$ Humberto Gómez ${ }^{3}$

\begin{abstract}
RESUMEN
Los humedales urbanos son sitios de alta biodiversidad pero que pueden estar bajo presión antrópica si no son bien manejados. En la ciudad de Punta Arenas (53ㅇ), el humedal de Tres Puentes posee características únicas para conformar un sitio con todos los componentes de conservación (educación, investigación, desarrollo sostenible). Se reporta su composición y abundancia en especies de aves acuáticas durante 6 temporadas estivales. Se registraron 31 especies lo que corresponde al $50 \%$ de las aves acuáticas continentales de la región de Magallanes. Se discute la variabilidad interanual y los posibles impactos producidos por el hombre.
\end{abstract}

Palabras clave: Aves acuáticas, humedal urbano Tres Puentes, conservación, Magallanes.

ABSTRACT

Urban wetlands are sites with high biodiversity but with anthropic pressure if not well manage. In Punta Arenas city $\left(53^{\circ} \mathrm{S}\right)$, Tres Puentes is a unique wetland to create a site with all conservation components (education, research, sustainable development). We report Waterfowl species composition and abundance during 6 breeding season is reported. There were 31 species, corresponding $50 \%$ of continental waterfowl of Magallanes region. Interannual variability in species richness, abundance and possible anthropic impacts are discussed.

Key words: Waterbirds, urban wetland, conservation, Magallanes. 


\section{INTRODUCCIÓN}

Los humedales, caracterizados por albergar una alta biodiversidad, son reconocidos por los múltiples beneficios hacia el ser humano como soporte de nutrientes, actividades recreativas y educación, por lo que juegan una función relevante para las estrategias de desarrollo socioeconómico sostenible (Tabilo 2003). En el extremo sur de Chile existen numerosos humedales continentales, principalmente en el ecosistema de estepa patagónica, donde existe una gran diversidad de aves acuáticas, pero que han sido escasamente estudiadas en hábitats prácticamente prístinos (e.g. Markham 1971, Jory et al.1974, Garay et al. 1991, Gibbons et al. 2007).

Por otro lado, los humedales urbanos aportan diversidad biológica y estética al paisaje incluso teniendo en cuenta los impactos que genera el desarrollo de las ciudades.

Sin embargo, la urbanización es una de las principales fuentes de deterioro ambiental que genera homogeneización de la biota (Klepel et al. 2004, Pouchard et al. 2006) debido a la falta de terreno disponible de bajo costo para la construcción (Kentula et al. 2004), siendo los impactos negativos para la biodiversidad aquellos que disminuyen la capacidad de carga del hábitat principalmente por fragmentación, cambios en la vegetación e introducción de especies invasoras (Dowd 1992, Robinson \& Cranswick 2003, Chace \& Walsh 2004, Burton 2007) y para el caso de la avifauna también aspectos de comportamiento y poblacionales (Burton et al. 2002b).

En la ciudad de Punta Arenas (53ㅇ) existe un humedal que se ha formado en una cuenca donde llega al aporte de agua por el drenaje de las colinas que lo rodean y por el aporte de agua lluvia (Harambour 2007)1. Este sitio ha sido modificado para urbanización desde hace al menos 30 años y recientemente por la construcción de vías de tránsito vehicular. El objetivo del estudio es la caracterización de la riqueza y abundancia de los ensambles de aves acuáticas que utilizan el humedal durante el período estival en años donde se ha modificado sucesivamente el hábitat.

\footnotetext{
Harambour, F. 2007. Sustentabilidad hídrica del humedal de Tres Puentes, Punta Arenas. Informe Final para CONAMA, XII Región.
}

\section{MATERIALES Y MÉTODOS}

El área de estudio comprende un humedal localizado dentro del área urbana de la ciudad de Punta Arenas $\left(53^{\circ} \mathrm{S}\right)$ en la región de Magallanes, extremo sur de Chile. El sitio se encuentra rodeado por viviendas, instalaciones industriales, una zona de relleno con áridos y un área verde correspondiente a un parque cementerio. A partir del año 2003, el humedal fue dividido en dos por una vía de tránsito vehicular y otras dos vías seccionaron la parte sur el año 2005. En el humedal se reconocen como tipos de hábitat un parche de bosque de ñirre (Nothofagus antarctica) con suelo pantanoso, vegas y áreas pantanosas con pastizal, tres espejos de agua permanentes y varias pequeñas lagunas temporales que varían en tamaño, algunas de las cuales se secan a mediados de verano.

Se censaron las aves acuáticas durante los períodos estivales entre los años 1995 - 1998 y 2004 - 2007. En cada período se efectuaron al menos dos censos mensuales por dos observadores, desde puntos aventajados de observación, entre septiembre y principios de marzo de cada temporada reproductiva, utilizándose binoculares 10x42 y telescopio 20-45x $y$ se registraron datos de especies que nidificaron en el área de estudio. La riqueza de especies se obtuvo para cada temporada como el número observado de especies registradas y también se calculó la riqueza mediante el estimador Chao 1 y número de Hill. Además, se calculó el índice de diversidad de Shannon - Wienner ( $\mathrm{H}^{\prime}$ ) y equidad de Pielou (J'). La abundancia se analizó en primer lugar agrupando los datos por especies en dos períodos: el primero correspondió a las temporadas iniciales de urbanización del humedal (1995 a 1998) y el siguiente a la fase de mayor alteración del humedal (2004 a 2007). Debido a que los datos de abundancia son multinormales y las muestras son dependientes, se comparó ambos períodos mediante la prueba no paramétrica de comparación de pares de Wilcoxon. La similitud porcentual de la riqueza de especies observada se obtuvo mediante el procedimiento de Schluter \& Ricklefts (Moreno 2001). Para observar el grado de similitud entre todas las temporadas reproductivas los datos de abundancia fueron transformados por $\log (\mathrm{x}+1)$ para formar una matriz de similitud con el índice de Bray - Curtis, con la que se realizó un análisis de conglomerados. 


\section{RESULTADOS}

En las seis temporadas de censos se registró en total 31 especies de aves acuáticas, representadas por seis familias (Apéndice I). El período de inicio de urbanización (1995-1998) dio cuenta de 26 especies, y entre cada una de las temporadas estivales la similitud fue de 51\%. Durante la máxima actividad de urbanización (2004-2007) se registraron 30 especies y entre cada una de las temporadas de censos la similitud fue de 43\%. Entre ambos períodos se encontró una similitud del 55\%. Las especies que se encontraron sólo en un período fueron Coscoroba coscoroba en las temporadas 1995-1998, y Anas versicolor, Netta peposaca, Tringa flavipes, Charadrius falcklandicus y Numenius phaeopus en las temporadas 2004-2007. Los mayores valores en la cantidad de especies observadas, estimaciones a través del índice Chao 1 , número de Hill y la diversidad específica y equidad ocurrieron en las temporadas estivales entre los años 2004 y 2007 (Tabla 1).

Las especies más abundantes fueron Anas georgica, Anas flavirostris y Chloephaga poliocephala. Se observó mayor influencia de Anas platalea sólo durante el período 2004 - 07 (Fig. 1). Durante las temporadas de los años 1995-98 las especies dominantes entregaron el 53,7\% de la abundancia media. En las temporadas de los años 2004-07 las especies dominantes entregaron 46,9\% de la abundancia media. La abundancia total entre períodos no difiere significativamente $(z=1,94$; $p=0,052$ ), pero se encontró que los ensambles de aves acuáticas de las diferentes temporadas se separan al 75\% de similitud (Fig. 2). La diversidad de Shannon - Wienner varió entre 0,89 y 1,05 bits/ind., y fue mayor en las temporadas de los años 2004-07.

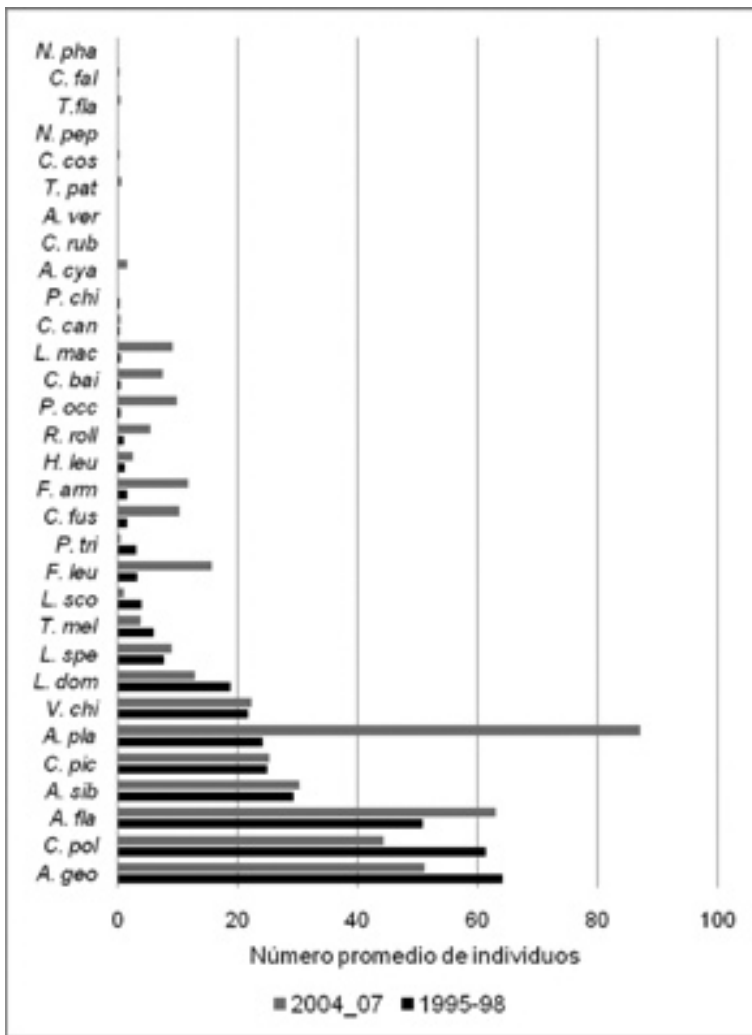

Fig. 1. Abundancia de aves acuáticas en el humedal de Tres Puentes. Barras negras corresponden al periodo 1995 - 98 y barras grises al periodo $2004-07$.

\section{DISCUSIÓN}

Comunidades de aves acuáticas en el humedal de Tres Puentes.

La avifauna acuática de la región de Magallanes es conocida por su distribución general (e.g. Venegas \& Jory 1979, Humphrey et al. 1970) pero existe desconocimiento por la abundancia y riqueza

TABLA 1. Valores de riqueza de especies (S) de aves acuáticas observadas durante los censos, estimaciones de riqueza por los índices Chao 1 y número de Hill (N1); diversidad según Shannon - Wienner (H') y equidad (J').

\begin{tabular}{cccccccc}
\hline temporada & $\mathrm{N}$ & S promedio & rango & Chao 1 & $\mathrm{~N} 1$ & $\mathrm{H}^{\prime}$ & $\mathrm{J}$ \\
\hline $1995-96$ & 8 & 14,88 & $12-19$ & 19,8 & 11,93 & 2,48 & 0,78 \\
$1996-97$ & 7 & 10,88 & $6-14$ & 26 & 8,75 & 2,17 & 0,74 \\
$1997-98$ & 11 & 10,91 & $8-15$ & 29,2 & 7,8 & 2,05 & 0,71 \\
$2004-05$ & 10 & 17,4 & $9-21$ & 30,2 & 13,17 & 2,58 & 0,8 \\
$2005-06$ & 6 & 14 & $11-16$ & 31 & 11,22 & 2,42 & 0,79 \\
$2006-07$ & 5 & 17,8 & $15-20$ & 31 & 12,52 & 2,53 & 0,74 \\
\hline
\end{tabular}




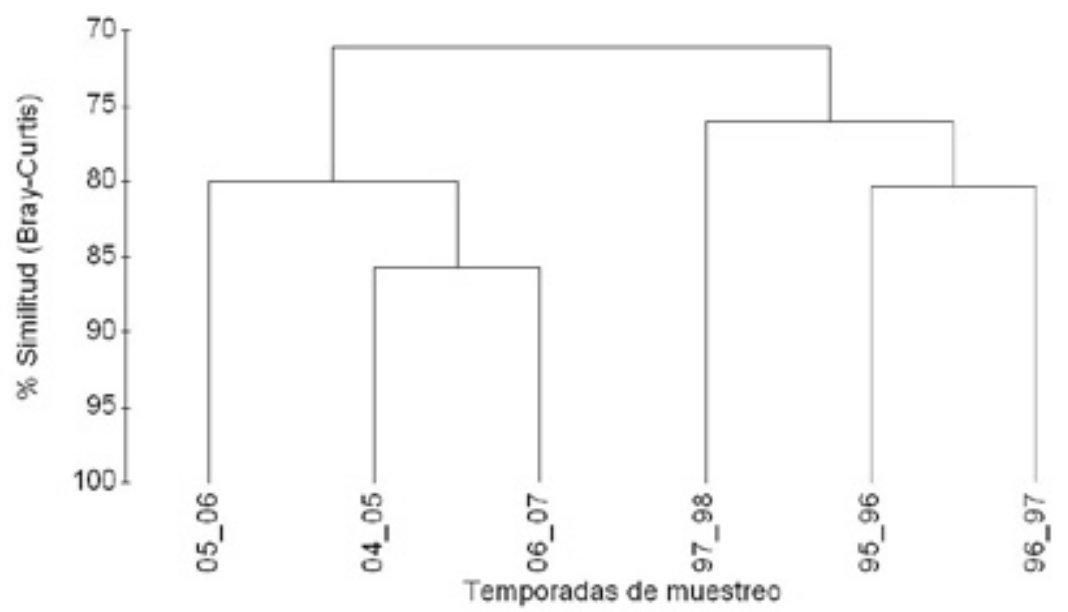

Fig. 2. Gráfico de similitud de Bray - Curtis usando la abundancia por especies en cada temporada estival.

en localidades específicas pese a la gran cantidad de humedales existentes en el ecosistema de estepa patagónica (e.g. Jory et al. 1974, Gibbons et al. 2007). Hasta ahora, la riqueza de aves acuáticas registradas en el humedal de Tres Puentes corresponde al 74\% de las aves acuáticas de Magallanes que frecuentan aguas interiores (Venegas \& Sielfeld 1998).

En el humedal de Tres Puentes, la riqueza de especies y el índice de diversidad fueron superiores en el período 2004-07. Debido a que el estimador Chao 1 se basa en el número de especies raras (Moreno 2001) los valores encontrados son casi el doble de lo observado. Esto concuerda con el registro de especies poco frecuentes o de distribución restringida a ambientes esteparios de Magallanes, como el caso de Netta peposaca (octubre de 2004), o Chloephaga rubidiceps. De esta última se observó grupos de 4 a 8 ejemplares, lo que además muestra que el humedal puede servir como un sitio de parada durante la migración pre y post reproductiva (Blanco et al. 2006), idea reforzada por el registro de ejemplares que fueron anillados en el sector de río San Juan (R. Matus, com. per.). También se asocia con la presencia de especies oportunistas de la disponibilidad de hábitat, debido a que se observaron fluctuaciones del nivel de agua que dejaron áreas pantanosas que fueron usadas por playeros y chorlos.

En función de valores de diversidad, el humedal de Tres Puentes es similar a lo descrito en humedales esteparios en el Parque Nacional Torres del Paine donde se encontró mayor diversidad en áreas con mayor heterogeneidad de hábitat (Garay et al. 1991). De igual forma se puede plantear que el aumento de la diversidad de aves acuáticas en el humedal de Tres Puentes, durante el período 2004 - 2007, se relacionaría con las fluctuaciones del nivel de agua y la riqueza de hábitat con diferentes características de vegetación.

Posibles efectos de la urbanización.

El humedal de Tres Puentes es un área sin protección oficial donde se han producido disturbios antrópicos de gran magnitud pero de corta duración y de forma interanual. Hasta el año 1995 se realizaron rellenos de áreas pantanosas y cuerpos de agua, lo que seguramente se reflejó en la disminución de aves hasta la temporada 1997-98, en que se observó un incremento de riqueza y abundancia de especies. Otras modificaciones comenzaron el año 2003 y se prolongaron hasta el 2006, con la disección del humedal por vías de tránsito vehicular.

En los períodos en que se alteraron los cuerpos de agua del humedal, la riqueza de especies y la abundancia no es menor a los meses sin perturbación, lo que sugiere una rápida adaptación de las aves a los cambios producidos en el hábitat. Sin embargo, existió variabilidad de la abundancia durante la última temporada, lo que podría reflejar el uso del hábitat de manera dinámica y de acuerdo a la disponibilidad de recursos. Esto se debió probablemente a que cuando se instala la vía de tránsito vehicular se produce un disturbio espacialmente restringido que 
no afecta la territorialidad de las aves (Burton et al. 2002a), lo que se refuerza por la presencia de aves que nidifican en el sitio.

La modificación de cuerpos de agua por urbanización genera alteración del ciclo hidrológico, variación en profundidad del agua y cambios en la estructura y composición de la vegetación (Kentula et al. 2004, Torres et al. 2006, Hierl et al. 2007). Para las aves, la perturbación sostenida también produce cambios conductuales afectando el éxito reproductivo, tiempo de alimentación o la tolerancia a la presencia humana (Milton et al. 1958, Burton et al. 2002a, 2002b, Steen et al. 2006); sin embargo, las aves pueden adaptarse a la presencia de la actividad humana si existe un manejo adecuado del hábitat (Riffell et al. 1996). Ambas respuestas de las aves dependen de la función que se quiere hacer del lugar y las medidas de conservación. En el humedal de Tres Puentes el avance de la urbanización no provocó cambios significativos en la abundancia total de especies y la composición de la avifauna acuática es levemente diferente entre los períodos estudiados, probablemente por la formación de una mayor heterogeneidad de hábitat.

Se ha demostrado que humedales de menor tamaño son de extrema importancia para la conservación de biodiversidad porque existe entre ellos conectividad para especies de amplio desplazamiento como las aves, siendo éste un elemento fundamental para la toma de decisiones sobre conservación (Gibbs 2000). Los cambios en el hábitat y la resiliencia de las aves acuáticas muestran que el humedal de Tres Puentes es un sitio de importancia regional para la conservación, tanto como refugio para las aves que llegan a reproducirse o como sitio de alimentación temporal para especies en tránsito a otras localidades. Además se debe considerar su alto potencial como sitio de educación ambiental por estar en un centro poblado. Por las mismas razones se recomienda mejorar las condiciones del humedal a través de restauración ecológica del hábitat (e.g. Grayson et al. 1999, Seigel et al. 2005) y monitorear las poblaciones de aves acuáticas en un contexto más amplio donde se incluya la dinámica hidrológica del humedal y la disponibilidad de hábitat, ya que la pérdida de biodiversidad puede no ser detectada sino hasta varios años después de los disturbios provocados por urbanización (Findlay \& Bourdages 2000).

\section{AGRADECIMIENTOS}

Agradecemos especialmente al Fondo de Protección Ambiental de CONAMA que ha financiado parte del trabajo en el humedal de Tres Puentes. A un revisor anónimo por las sugerencias hechas al manuscrito. Y a IDEAWILD que proporcionó equipo de terreno que actualmente se está usando en la conservación y educación ambiental de las aves del humedal de Tres Puentes.

\section{LITERATURA CITADA}

Blanco, D.E., R. Matus, O. Blank, V.M. de la Balze, \& S.M. Zalba 2006. The Ruddy-headed Goose Chloephaga rubidiceps mainland population: a flyway perspective. In G.C. Boere, C.A. Galbraith \& D.A. Stroud (Eds.) Waterbirds around the world, pp. 195-196. The Stationery Office, Edinburgh, UK.

Burton, N. H. K. 2007. Landscape approaches to studying the effects of disturbance on waterbirds. Ibis 149: 95 - 101.

Burton, N. H. K., M. J. S. Armitage, A. J. Musgrove \& M. M. Rehfish 2002a. Impacts of man-made landscape features on numbers of estuarine waterbirds at low tide. Environmental Management 30: 857 - 864 .

Burton, N. H. K., M. M. Rehfish \& N. A. Clark 2002b. Impacts of disturbance from construction work on the densities and feeding behavior of waterbirds using the intertidal mudflats of Cardiff bay, UK. Environmental Management 30: $865-871$.

Chace, J. F. \& J. J. Walsh 2004. Urban effects on native avifauna: a review. Landscape and Urban Planning 1 - 24.

Dowd, C. 1992. Effect of development on bird species composition of two urban forested wetlands in Staten Island, New York. Journal of Field Ornithology 63: $455-461$.

Findlay, C. S. \& J. Bourdages 2000. Response time of wetland biodiversity to road construction on adjacent lands. Conservation Biology 14: $86-94$.

Garay, G., W. E. Johnson \& W. L. Franklin 1991. Relative abundance of aquatic birds and their use of wetlands in the Patagonia of southern 
Chile. Revista Chilena de Historia Natural 64: $127-137$.

Gibbs, J. P. 2000. Wetland loss and biodiversity conservation. Conservation Biology 14: $314-317$.

Gibbons, J., Y. A. Vilina \& J. Cárcamo 2007. Distribución y abundancia de cisne coscoroba (Coscoroba coscoroba), Cisne de cuello negro (Cygnus melancoryphus) y del Flamenco chileno (Phoenicopterus chilensis) en la región de Magallanes. Anales Instituto Patagonia (Chile) 35: 53 - 58.

Grayson, J. E., M. G. Chapman \& A. J. Underwood 1999. The assessment of restoration of habitat in urban wetlands. Landscape and Urban Planning 43: 227 - 236.

Hierl, L. A., C. S. Loftin, J. R. Longcore, D. G. McAuley \& D. L. Urban 2007. A multivariate assessment of changes in wetland habitat for waterbirds at Moosehorn National Wildlife Refuge, Maine, USA. Wetlands 27: 141 - 152.

Humphrey, P. S., D. Bridge, P. W. Reynolds \& R. T. Peterson 1970. Birds of Isla Grande (Tierra del Fuego). Preliminary Smithsonian Manual. Lawrence, Kansas.

Jory, J. E., C. Venegas \& W. Texera 1974. La avifauna del Parque Nacional Laguna de los Cisnes, Tierra del Fuego, Chile. Anales Instituto Patagonia (Chile) 5: $131-154$.

Kentula, M. E., S. E. Gwin \& S. M. Pierson 2004. Tracking changes in wetlands with urbanization: sixteen years of experience in Portland, Oregon, USA. Wetlands 24: $734-743$.

Kleppel, G. S., S. A. Madewell, \& S. E. Hazzard 2004. Responses of emergent marsh wetlands in upstate New York to variations in urban typology. Ecology and Society 9: 1. [online] URL: http://www.ecologyandsociety. org/vol9/iss5/art1

Markham, B. 1971. Censo invernal de cisnes y flamencos en Magallanes. Anales Instituto Patagonia (Chile) 2: 146 - 157

Milton, W. W., B. H. Wingfield \& J. B. Low 1958. Effects of habitat deterioration on bird populations of a small Utah marsh. Condor 60: $220-226$.
Moreno, C. 2001. Métodos para medir la biodiversidad. M \& T Manuales y Tesis SEA, Vol. 1, Zaragoza.

Pouchard, A., M. Aguayo, E. Peña \& R. Urrutia 2006. Multiple effects of urbanization on the biodiversity of developing countries: The case of a fast-growing metropolitan area (Concepción, Chile). Biological Conservation 127: $272-281$

Riffell, S. K., K. J. Gutzwiller \& S. H. Anderson 1996. Does repeated human intrusion cause cumulative declines in avian richness and abundance? Ecological Applications 6: 492 $-505$.

Robinson J. A. \& P. A. Cranswick 2003. Large-scale monitoring of the effects of human disturbance on waterbirds: a review and recommendations for survey design. Ornis Hungarica 12-13: 199 - 207.

Seigel, A., C. Hatfield \& J. M. Hartman 2005. Avian response to restoration of urban tidal marshes in the Hackensack Meadowlands, New Jersey. Urban Habitats 3: 87 - 116.

Steen, D. A., J. P. Gibbs \& S. T. A. Timmermans 2006. Assessing the sensitivity of wetland bird communities to hydrologig change in the eastern Great Lakes region. Wetlands 26: $605-611$.

Tabilo, E. 2003. El beneficio de los humedales en la Región Neotropical. Centro Neotropical de Entrenamiento en Humedales, La Serena - Chile.

Torres, M., Z. Quinteros \& F. Takano 2006. Variación temporal de la abundancia y diversidad de aves limícolas en el Refugio de Vida Silvestre Pantanos de Villa, Lima - Perú. Ecología Aplicada 5: 119 - 125.

Venegas, C. \& J. Jory 1979. Guía de campo para las aves de Magallanes. Publicaciones del Instituto de la Patagonia, Serie Monografías $\mathrm{N}^{\circ}$ 11, Punta Arenas (Chile) 253 pp.

Venegas, C. \& W. Sielfeld 1998. Catálogo de los vertebrados de la región de Magallanes y Antártica chilena. Ediciones Universidad de Magallanes, Punta Arenas (Chile) 122 pp. 
APÉNDICE. Lista de especies de aves acuáticas registradas en el humedal de Tres Puentes durante los censos de los años 1995 - 1998 y 2004 - 2007. Especies que anidan en el humedal se marcan con *. Fuera de los censos se registró nidificando Gallinago paraguaiae y registros esporádicos de Gallinago stricklandii.

\begin{tabular}{|c|c|c|}
\hline Nombre común & Nombre científico & Residencia en el humedal \\
\hline Pimpollo & Rollandia rolland & Residente con pocos ejemplares en invierno* \\
\hline Blanquillo & Podiceps occipitalis & Residente estival* \\
\hline Bandurria & Theristicus melanopis & Residente con pocos ejemplares en invierno \\
\hline Flamenco chileno & Phoenicopterus chilensis & Ocasional \\
\hline Coscoroba & Coscoroba coscoroba & Ocasional \\
\hline Canquén & Chloephaga poliocephala & Residente, con pocos ejemplares en invierno \\
\hline Caiquén & Chloephaga picta & Residente, con decenas de ejemplares en invierno* \\
\hline Canquén colorado & Chloephaga rubidiceps & Frecuente en bajas cantidades \\
\hline Quetru volador & Tachyeres patachonicus & Residente* \\
\hline Pato juarjual & Lophonetta specularioides & Residente con pocos ejemplares en invierno* \\
\hline Pato jergón chico & Anas flavirostris & Residente con pocos ejemplares en invierno* \\
\hline Pato capuchino & Anas versicolor & Ocasional \\
\hline Pato real & Anas sibilatrix & Residente estival* \\
\hline Pato jergón grande & Anas georgica & Residente estival* \\
\hline Pato colorado & Anas cyanoptera & Ocasional \\
\hline Pato cuchara & Anas platalea & Residente estival* \\
\hline Pato negro & Netta peposaca & Raro, solo dos ejemplares en diciembre del 2004 \\
\hline Tagua común & Fullica leucoptera & Residente \\
\hline Tagua chica & Fullica armillata & Residente* \\
\hline Queltehue & Vanellus chilensis & Residente estival* \\
\hline Pilpilén austral & Haematopus leucopodus & Residente estival frecuente pero con pocos individuos* \\
\hline Pitotoy chico & Tringa flavipes & Ocasional \\
\hline Pitotoy grande & Tringa melanoleuca & Ocasional \\
\hline Chorlo de doble collar & Charadrius falcklandicus & Frecuente, dependiendo del nivel del agua \\
\hline Chorlo chileno & Charadrius modestus & Ocasional, dependiendo del nivel del agua \\
\hline Playero de Baird & Calidris bairdii & Frecuente, dependiendo del nivel del agua \\
\hline Playero de lomo blanco & Calidris fuscicollis & Frecuente, dependiendo del nivel del agua \\
\hline Playero ártico & Calidris canutus & Raro, solo registros en las temporadas 1997/98 \\
\hline Pollito de mar tricolor & Phalaropus tricolor & Ocasional \\
\hline Gaviota dominicana & Larus dominicanus & Común y abundante en invierno \\
\hline Gaviota austral & Larus scoresbii & Ocasional \\
\hline Gaviota cahuil & Larus maculipenis & Común y abundante a fines de verano \\
\hline Zarapito común & Numenius phaeopus & Raro, solo registro en temporada 2004 \\
\hline
\end{tabular}


A. $\mathrm{KUSCH}$ et al. 